\title{
Jung, fremd und gefährlich? - Migration und Jugendkriminalität
}

Christian Walburg

$\mathrm{B}$ undesweit verfügt heute mehr als jeder vierte Jugendliche über eigene oder familiäre Migrationserfahrungen, in vielen Ballungsräumen stellen junge Einwanderer und Nachkommen von Einwanderern gar 40\% und mehr der altersgleichen Bevölkerung. Jugendliche mit Migrationshintergrund gelten in vielen klassischen wie neueren Einwanderungsländern als die eigentliche Problemgruppe, wenn es um delinquentes und insbesondere gewaltsames Verhalten unter jungen Menschen geht. In diesem Personenkreis vereinen sich Merkmale, die bereits jeweils für sich genommen ein besonderes Bedrohungspotenzial suggerieren: Zu Vorbehalten gegenüber der halbstarken, orientierungslosen und deshalb gefährlichen Jugend gesellen sich Ängste gegenüber dem Unbekannten und Fremden, der das gesellschaftliche Zusammenleben bedroht. Die generalisierende Gleichsetzung von Jugend und Fremdheit auf der einen und Delinquenz auf der anderen Seite hilft, die Komplexität des Phänomens Kriminalität zu reduzieren. Die Verknüpfung von Kriminalität mit Einwanderern und anderen, in der Regel sozial schwächeren Gruppen trägt aber auch dazu bei, das Bedürfnis nach einem Bild von Kriminalität als etwas Außergewöhnlichem zu befriedigen, als etwas, das mit „uns“, dem „Normalbürger“, nichts zu tun hat. Mit Elias und Scotson (1990) lässt sich die Vorstellung größerer Rohheit, Disziplin- und Gesetzlosigkeit unter Fremden überdies als Paradefall des „universal-menschlichen Themas" einer vermeintlichen Überlegenheit von Angehörigen der wie auch immer definierten Eigengruppe gegenüber Nichtmitgliedern interpretieren. Die negativere Beurteilung von Fremdgruppen erscheint dann als Ausdruck eines Bedürfnisses nach einem positiven Selbstkonzept; die Stigmatisierung der Neuankömmlinge ist zugleich aber auch Mittel zum Erhalt der eigenen Vorrangstellung.

In Deutschland lässt sich die Diskussion um besondere Kriminalitätsrisiken unter den Nachkommen der Arbeitsmigranten bis in die Mitte der siebziger Jahre zurückverfolgen. Seit dieser Zeit waren sie, Jugendliche aus Familien aus dem Mittelmeerraum, in der Regel gemeint, wenn von „der Ausländerkriminalität" die Rede war. Später wurde dieses Bild, das zu allen Zeiten auch Bestandteil der jeweiligen Debatten um die Einwanderungs- und Flüchtlingspolitik war, ergänzt durch den Bereich grenzüberschreitender (Organisierter) Kriminalität sowie durch Befürchtungen einer durch Flüchtlinge und Asylsuchende „importierten Kriminalität“. In den letzten Jahren rückten indes wieder hier lebende Jugendliche ausländischer Herkunft in den Mittelpunkt der Diskussion. Neben den (vor allem türkischstämmigen) Gastarbeiternachkommen, die zusätzlich zunehmend unter den Verdacht einer Hinwendung zu radikal-islamischen Überzeugungen gerieten, nahm man nun auch die in jüngerer Zeit und vorwiegend noch selbst immigrierten jungen (Spät-)Aussiedler sowie Jugendliche aus Flüchtlingsfamilien aus Südosteuropa oder dem Nahen Osten in den Blick. Aktuell ließen die gewaltsamen Unruhen unter Einwandererjugendlichen in Pariser und anderen französischen Vorstädten im Herbst 2005 Befürchtungen einer ähnlichen Entwicklung in Deutschland aufkommen. Im Frühjahr 2006 bestimmte dann ein Brief, mit dem Lehrer einer Berliner Hauptschule wegen Gewaltproblemen an ihrer Schule an die Öffentlichkeit gegangen waren, über Wochen das öffentliche Bild von jungen Migranten in Deutschland. Die Schule wird weit überwiegend von Jugendlichen aus Einwanderer- und insbesondere Flüchtlingsfamilien besucht. Dies alles spielt sich ab vor dem Hintergrund einer Entwicklung, bei der wieder deutlicher Grenzen der Zugehörigkeit markiert und Migranten verstärkt als „ethnisch Fremde“ betrachtet werden. Damit korrespondierend werden Kriminalität und Gewalt derzeit weniger in Verbindung mit sozialen Konflikten, sondern vor allem mit kulturellen Defiziten diskutiert.

Dieser Beitrag soll einen Überblick über den aktuellen Forschungsstand zur Verbreitung von Delinquenz unter jungen Migranten im Hell- und Dunkelfeld geben. In einem weiteren Schritt soll die Reichweite und empirische Bewährung von Erklärungsansätzen zu Kriminalität unter Jugendlichen mit Migrationshintergrund erörtert werden.

\section{Werden junge Migranten häufiger wegen einer Straftat registriert?}

Ausgangspunkt vieler Betrachtungen und insbesondere des öffentlichen Diskurses über
Kriminalität sind die offiziellen Kriminalstatistiken, die Auskunft erteilen über die Zahl der von der Polizei bzw. Justiz registrierten Tatverdächtigen, Angeklagten, Verurteilten und Inhaftierten, das heißt über das Hellfeld der Kriminalität. Für Aussagen über die Verbreitung von delinquentem Verhalten unter jungen Migranten (und nicht nur über die Häufigkeit der offiziellen Registrierung) sind Kriminalstatistiken schon deshalb nur begrenzt geeignet, weil die darin aufgeführte Zahl an Straftätern das Ergebnis eines gesellschaftlichen Definitionsprozesses ist. Ein Vergleich der Verbreitung delinquenten Verhaltens unter Jugendlichen ausländischer Herkunft und unter jungen Einheimischen auf Basis der Polizeilichen Kriminalstatistik, der Strafverfolgungs- oder Strafvollzugsstatistik setzt daher voraus, dass ein bestimmtes Verhalten in beiden Gruppen mit gleicher Wahrscheinlichkeit von den jeweils zuständigen Stellen als Straftat definiert wird. Dass aber junge Migranten, zumal wenn sie im Straßenbild eher auffallen und bestimmte Stereotype bedienen, ein erhöhtes Risiko aufweisen, kontrolliert $\mathrm{zu}$ werden, ist zumindest nicht unplausibel, wenn auch gesicherte Erkenntnisse hierzu rar sind. Dagegen liefern auf Opferbefragungen beruhende Analysen zum Anzeigeverhalten gegenüber Migranten deutliche Hinweise für erhöhte Kontrollrisiken in dieser Gruppe. Die Entscheidung über eine Anzeige wird danach in gewissem Maße auch durch die Zuordnung des Täters zu einer als fremdethnisch definierten Gruppe bestimmt (Oberwittler et al. 2001, S. 32 ff.; Wilmers et al. 2002, S. 34 ff.; Mansel \& Albrecht 2003). Hierin könnten sich negative Einstellungen gegenüber Einwanderern bemerkbar machen. Zudem dürfte sich auswirken, dass in diesen Konstellationen seltener ein persönliches Näheverhältnis zwischen Opfer und Täter besteht, welches die Fähigkeit zur informellen Konfliktregulierung positiv beeinflusst. Nicht übersehen werden darf dabei jedoch, dass eine verstärkte Anzeigegeneigtheit von Einheimischen gegenüber Migranten jedenfalls zum Teil durch eine größere Zurückhaltung beim Anzeigeverhalten in „innerethnischen“ Konfliktsituationen (d.h. bei Auseinandersetzungen zwischen Migranten derselben Herkunft) kompensiert werden könnte. 
Doch auch eine Aussage über die Registrierungshäufigkeit von Jugendlichen ausländischer Herkunft und ein entsprechender Vergleich mit jungen Einheimischen ist durch einen Blick in offizielle Kriminalstatistiken nicht ohne Weiteres möglich, denn diese geben gar keine Auskunft über die absolute oder relative, das heißt in das Verhältnis zu dem entsprechenden Bevölkerungsanteil gesetzte Zahl der wegen einer Straftat registrierten jungen Migranten oder Nachkommen von Migranten. Zwar werden die registrierten Personen nach ihrer Staatsangehörigkeit unterteilt, es lässt sich also angeben, wie viele der jungen Tatverdächtigen, Verurteilten und Inhaftierten nicht den deutschen Pass besitzen. Auch wenn der Anteil der registrierten ausländischen Jugendlichen in allen drei Bereichen seit Mitte der 90er Jahre insgesamt kontinuierlich und deutlich zurückgegangen ist, sticht ihr Anteil insbesondere bei Gewaltdelikten weiterhin hervor (vgl. zum Ganzen Walburg 2007, 241 ff.). Jedoch kann von der Gruppe der in den Kriminalstatistiken erfassten Ausländer nur sehr bedingt auf die Zahl der wegen einer Straftat registrierten Migranten geschlossen werden. So gehören zu den etwa in der PKS registrierten Ausländern Personen, die (als Touristen oder Durchreisende) gar keine Migranten sind. Andere sind zwar (als Personen ohne legalen Aufenthalt) nach Deutschland immigriert, dürfen aber bei der Berechnung der Kriminalitätsbelastung der inländischen Wohnbevölkerung mit Zuwanderungsgeschichte ebenfalls nicht berücksichtigt werden, da sie nicht hier gemeldet sind.

Vor allem aber hat heute auch ein erheblicher Teil der Jugendlichen mit deutschem Pass eigene oder familiäre Migrationserfahrungen. Mit der erstmaligen Erfassung migrationsspezifischer Daten im Mikrozensus 2005 liegen hierzu nun recht präzise Zahlen vor: Danach sind bundesweit rund 10\% der unter 25jährigen Ausländer; weitere 17,3\% sind Deutsche, haben aber ebenfalls eigene oder familiäre Migrationserfahrungen (Konsortium Bildungsberichterstattung 2006, 142 ff.). Der größte Teil davon sind eingebürgerte Migranten der 1 . und vor allem 2 . Generation, darunter viele Nachkommen von Migranten aus den ehemaligen Gastarbeiter-Anwerbeländern. Hinzu kommen Kinder aus binationalen Ehen sowie die zwischen Ende der 80er und Mitte/ Ende der 90er Jahre stark angewachsene Gruppe der jungen (Spät-)Aussiedler, welche mit ihrem Zuzug nach Deutschland ebenfalls deutsche Staatsangehörige wurden. Dass eine Unterteilung der Jugendlichen anhand ihrer Staatsangehörigkeit im Hinblick auf das soziale Phänomen der Migration nur noch wenig sinnvoll ist, zeigt auch ein Blick auf die Selbstbe- richte unter Duisburger Jugendlichen: In dieser Stadt mit einem besonders hohen Anteil türkischstämmiger Einwohner gab in einer Schülerbefragung im Jahr 2005 etwa jede(r) dritte Zehntklässler(in) türkischer Herkunft an, die deutsche Staatsangehörigkeit zu besitzen. Diese (deutschen) „Jugendlichen mit Migrationshintergrund" werden in Hellfeldstatistiken jedoch in aller Regel nicht gesondert erfasst, sondern gehen in die Gesamtgruppe der deutschen Jugendlichen ein. Eine Ausnahme hierzu bildet etwa die Berliner Polizeiliche Kriminalstatistik, die für das Jahr 2006 bezüglich der „Jugendgruppengewalt“ neben ausländischen Tatverdächtigen mit einem Gesamtanteil von 27,4\% weitere $17,4 \%$ als „deutsche Tatverdächtige nichtdeutscher Herkunft" ausweist (Polizeipräsident in Berlin 2007, 91 ff.). Ein entsprechender Bevölkerungsanteil zur Einordnung dieses Wertes wird jedoch nicht genannt.

Aus einer Sonderauswertung der „Intensivtäter"-Akten der Berliner Staatsanwaltschaft geht hervor, dass dort im Sommer 2005 knapp die Hälfte der von den Strafverfolgungsbehörden als besonders problematisch klassifizierten Tatverdächtigen nicht die deutsche Staatsangehörigkeit besaßen. Der Anteil der Personen mit Migrationshintergrund lag gar bei 70\%. Den Akten waren auch Hinweise auf die jeweiligen Migrationsumstände und -motive zu entnehmen: Dabei zeigte sich, dass es sich bei den Betroffenen nur zu einem kleineren Teil um Nachkommen klassischer Arbeitsmigranten handelte, im Vordergrund standen vielmehr Flucht- und Asylkonstellationen. Die jeweiligen Familien waren vielfach erst in den 90er Jahren auf der Suche nach Schutz vor Verfolgung und Instabilität, häufig aus dem Nahen Osten, nach Deutschland gekommen, in vielen Fällen waren die (ausländer-)rechtlichen und sozialen Lebensbedingungen als ausgesprochen prekär zu bezeichnen (Ohder \& Huck 2006, 11 ff.).

Auch die vielfach geäußerte Vermutung einer überdurchschnittlich häufigen Registrierung junger, v.a. männlicher (Spät-)Aussiedler lässt sich, da zu dieser Gruppe in offiziellen Kriminalstatistiken in aller Regel keine gesonderten Angaben gemacht werden (bzw. keine relativen, d.h. in das Verhältnis zur Bevölkerungsgröße gesetzte Belastungszahlen gebildet werden können), nur durch Sonderauswertungen überprüfen. Während sich die Rahmenbedingungen für die Neuankömmlinge seit den frühen 90er Jahren verschlechterten, deuten neuere Untersuchungen auf eine zum Teil recht deutliche Höherbelastung der in jüngerer Zeit eingewanderten (männlichen) jugendlichen Spätaussiedler im Hellfeld hin (so zu Niedersachsen Pfeiffer et al. 2005, 52 ff. und, zu
Baden-Württemberg bzw. Bayern, tendenziell bereits Grundies 2000, 302 ff. sowie Luff 2000, $82 \mathrm{ff}$.). Überdies beobachtete man bundesweit einen steigenden Anteil von (Spät-)Aussiedlerjugendlichen im Jugendstrafvollzug: Eine Untersuchung der Zusammensetzung der 1998 in den Jugendstrafanstalten der westlichen Bundesländer Inhaftierten ergab einen (Spät-)Aussiedleranteil von $10 \%$, der entsprechende Bevölkerungsanteil wurde auf 5,5\% geschätzt (Pfeiffer \& Dworschak 1999, 186). Für das Jahr 2002 ging man dann gar von einem Strafgefangenenanteil von 14\% (bei einem Bevölkerungsanteil von 6\%) aus (Kleimann \& Pfeiffer 2004, 383). Im Längsschnittvergleich zeigte sich in Bezug auf junge Türken im Übrigen ein gegenläufiger Befund: Lag deren Anteil in fünf untersuchten westdeutschen Jugendstrafanstalten im Jahr 1995 noch bei 19\%, so war er bis 2003 auf $7,1 \%$ zurückgegangen. Diese Abnahme ist wohl zum Teil auf in diesem Zeitraum zunehmende Einbürgerungen in dieser Migrantengruppe zurückzuführen, dies allein dürfte den Rückgang jedoch nicht erklären.

Insgesamt deuten die Untersuchungen des polizeilichen und justiziellen Hellfeldes weiterhin auf eine erhöhte Registrierung-, Verurteilungs- und Inhaftierungshäufigkeit von Jugendlichen mit Migrationshintergrund hin. Es zeigt sich allerdings, dass ein genaueres Hinschauen lohnt: Zum Einen überwiegt mittlerweile wohl die Einschätzung, dass eine pauschale Gegenüberstellung von Tatverdächtigen-, Verurteilten- oder Gefangenenraten deutscher und ausländischer Jugendlicher auch bei Berücksichtigung entsprechender Verzerrungsfaktoren kaum kriminologische Aussagekraft besitzt, da das Merkmal der eigenen oder familiären Migrationserfahrung anhand der Staatsangehörigkeit nicht adäquat erfasst wird. Zum Anderen handelt es sich bei den Jugendlichen mit Migrationshintergrund keineswegs um eine homogene Gruppe. Während ein Teil der erst vor kürzerer Zeit zugewanderten, vor allem männlichen Jugendlichen aus Flüchtlings- oder auch (Spät-)Aussiedlerfamilien in Analysen etwa zu sogenannten Intensivtätern oder Inhaftierten im Jugendstrafvollzug besonders auffällt, gibt es hinsichtlich der überwiegend in Deutschland geborenen und aufgewachsenen Nachkommen der klassischen Arbeitsmigranten durchaus auch Hinweise auf einen Rückgang der Registrierungshäufigkeit.

\section{Selbstberichtete Delinquenz unter jungen Migranten}

Seit einigen Jahren liegen nun vermehrt Informationen aus Dunkelfeldstudien zur Verbreitung von Delinquenz unter jungen Mig- 
ranten vor. Gegenüber Hellfeldzahlen haben diese auf Selbstberichten beruhenden Befunde den Vorteil, unabhängig von Bewertungsprozessen durch die Instanzen sozialer Kontrolle zu sein. Nichtsdestoweniger muss im Auge behalten werden, dass auch Täterbefragungen nur ein weiteres spezifisches, entscheidenden Grenzen unterworfenes Bild „der Kriminalität“ zeichnen. Hinsichtlich der Vergleichbarkeit der Selbstangaben von Jugendlichen mit und ohne Migrationshintergrund ist fraglich, ob diese im selben Maße durch Schülerbefragungen erreichbar sind und ob es nicht gewichtige Unterschiede im Antwortverhalten gibt. So kann möglicherweise nicht unbedingt von einem einheitlichen Verständnis und einer vergleichbaren Bewertung von Situationen ausgegangen werden, die von den Befragten unter die entsprechenden Fragen zu subsumieren sind. Auch die Bereitschaft, überhaupt (korrekte) Auskünfte zu eigenem strafbaren Verhalten zu erteilen, ist unter Umständen verschieden und dürfte davon abhängen, für wie attraktiv eine entsprechende Angabe in der Befragungssituation gehalten wird; hier sind sowohl eine größere Zurückhaltung als auch Übertreibungen denkbar.

Die vorliegenden Befunde aus Dunkelfeldstudien sind, da unterschiedliche Untersuchungsgebiete zugrunde liegen, die Stichprobenzusammensetzung und vor allem die Art der Gruppenbildung divergiert, nicht ganz vergleichbar. In einigen Untersuchungen sind zunächst schlicht die Angaben deutscher und ausländischer Jugendlicher gegenübergestellt worden. Zum Teil ergaben sich danach zwar erhöhte Täterraten ausländischer Jugendlicher gegenüber deutschen Befragten (Heitmeyer 1995, 405; Mansel \& Hurrelmann 1998, 97 ff.; Lösel \& Bliesener 2003, 58 ff.), die Unterschiede fielen jedoch regelmäßig geringer aus als in der Polizeilichen Kriminalstatistik. In der Bremer Längsschnittstudie - bei dieser sind nur Haupt- und Sonderschulabgänger befragt worden, sodass der Einfluss der Bildungsbeteiligung von Anfang an kontrolliert wurde - lagen die Anteile von jungen Ausländern und Deutschen hingegen auf vergleichbarem Niveau, in einigen Deliktsbereichen waren die nichtdeutschen Befragten dort sogar etwas niedriger belastet (Othold \& Schumann 2003).

Da jedoch Migrationserfahrungen in der Familie durch das Merkmal der Staatsangehörigkeit nicht (mehr) hinreichend präzise abgebildet werden, ist in jüngeren Untersuchungen darüber hinaus die Herkunft der Jugendlichen und ihrer Eltern erfasst worden. Auf diese Weise lassen sich Aussagen über die Verbreitung selbstberichteter Delinquenz unter jungen
Aussiedlern oder beispielsweise in der Gruppe der Jugendlichen mit türkischer Herkunft (ob mit oder ohne türkische Staatsangehörigkeit) treffen. Auch hier ergeben sich indes Definitionsprobleme, denn auch die Frage, wem ein "Migrationshintergrund" attestiert werden soll, unterliegt (in ihrer ausgrenzenden Wirkung im Übrigen nicht unproblematischen) gesellschaftlichen Konstruktionsprozessen. In sozialwissenschaftlichen Studien wird ein solcher häufig dann angenommen, wenn zumindest ein Elternteil im Ausland geboren wurde.

Nach den aktuellen Dunkelfelduntersuchungen ergibt sich hinsichtlich der Gesamtdelinquenzbelastung, die stark durch weit verbreitete, jugendtypische Bagatelldelikte geprägt ist, ein recht einheitliches Bild: Jugendliche mit Migrationshintergrund berichten insgesamt gesehen kaum häufiger delinquentes Verhalten als autochthone Jugendliche. Dasselbe gilt, betrachtet man einzelne Deliktsbereiche, für leichtere Eigentums- und Sachbeschädigungsdelinquenz (Naplava 2003, 75 ff.). Bezüglich des Ladendiebstahls werden, vor allem unter türkischstämmigen Mädchen, regelmäßig deutlich niedrigere Täteranteile als unter altersgleichen einheimischen Mädchen beobachtet (Boers et al. 2006, 80; Baier \& Pfeiffer 2007, 23).

Im Zentrum des Interesses steht jedoch die Frage, ob junge Migranten vermehrt die Begehung von Gewaltdelikten wie Körperverletzungen oder Raub (etwa das „Abziehen“ von Mitschülern) berichten. Im Rahmen der Schülerbefragungen des Kriminologischen Forschungsinstitutes Niedersachsen (KFN) in verschiedenen Großstädten gaben türkischstämmige Jugendliche und Befragte aus dem ehem. Jugoslawien in den Jahren 1998 und 2000 zum Teil bis zu zweimal häufiger als ihre einheimischen Mitschüler/innen an, ein Gewaltdelikt begangen zu haben. Einen ähnlichen Befund ergab die Auswertung der KFN-Befragungen in verschiedenen westdeutschen Städten und Regionen im Jahr 2005 sowie die Studie des Freiburger Max-Planck-Institutes in den Städten Köln und Freiburg im Jahr 1999. In diesen Untersuchungen fielen Migrantenjugendliche zudem mit zum Teil deutlich erhöhten Mehrfachtäteranteilen auf (Wilmers et al. 2002, 107 f.; Baier \& Pfeiffer 2007; Naplava 2003, 82 ff.). Dem hingegen berichteten die Duisburger Jugendlichen türkischer Herkunft zwischen 2002 und 2005 im Rahmen der Längsschnittstudie „Kriminalität in der modernen Stadt“ im 7. bis 10. Jahrgang kaum häufiger als ihre einheimischen Mitschüler die Begehung von Gewaltdelikten, und sie waren auch unter den Mehrfachtätern nicht überrepräsentiert (Boers et al. 2006, 79 ff.). Bei einer geschlechtsspezifischen
Betrachtung fielen besonders die tendenziell unterdurchschnittlichen Gewalttäterraten der türkischstämmigen Schülerinnen in Duisburg auf; in den Städten Hamburg, Hannover und München dagegen waren die weiblichen Befragten türkischer Herkunft mehr als doppelt so hoch mit Gewalt belastet wie einheimische Schülerinnen (Enzmann et al. 2004, 276).

Kein eindeutiges Bild einer vermehrten Beteiligung an delinquentem Verhalten lieferten die Studien entgegen manch öffentlichem Eindruck zunächst auch in Bezug auf junge (Spät)Aussiedler. Während von diesen Jugendlichen in einer Untersuchung in Städten und Gemeinden Nordrhein-Westfalens (Strobl \& Kühnel 2000, 155 ff.), in den Duisburger Schülerbefragungen (Boers et al. 2006, 80 f.) sowie in den ersten Erhebungen des KFN (Enzmann et al. 2004, 276) nicht oder allenfalls geringfügig vermehrt delinquentes Verhalten und insbesondere auch Gewaltdelikte berichtet wurde, fanden sich im Rahmen einer Schülerbefragung im Kreis Soest unter jungen Aussiedlern vor allem erhöhte Mehrfachtäteranteile (Mansel 2001, 84 ff.). In der bayerischen Untersuchung zu Gewalt im Schulkontext berichteten Aussiedlerjugendliche (ähnlich wie sonstige junge Migranten) ebenfalls vermehrt eigenes Gewalthandeln (Fuchs et al. 2005, 191 ff.). Hinweise auf erhöhte Gewaltdelinquenz unter jungen (Spät-)Aussiedlern fanden sich dann ebenfalls in der Münsteraner Teilstudie des Projektes „Kriminalität in der modernen Stadt“ (Walburg 2007, 252 ff.). Anders als in den vorangegangenen Befragungen waren diese schließlich auch in der Schülerbefragung des KFN im Jahr 2005 höher gewaltbelastet als einheimische Jugendliche. Die Autoren verwiesen hierbei auf die Möglichkeit einer „Normalisierung des Antwortverhaltens", d.h. der Aufgabe einer anfänglich größeren Zurückhaltung in dieser Gruppe mit zunehmender Aufenthaltsdauer. Die höhere Gewaltbelastung schlug sich auch in erhöhten Mehrfachtäteranteilen nieder: Männliche Jugendliche aus Russland und Polen berichteten mit 8,4\% bzw. 9,1\% nun rund zweimal so häufig wie ihre einheimischen Mitschüler $(4,1 \%)$, im vorangegangenen Jahr fünf und mehr Gewaltdelikte begangen zu haben. Die höchsten Anteile an Mehrfachtätern von Gewaltdelikten stellten in dieser Befragung aber weiterhin männliche Jugendliche türkischer Herkunft $(13,2 \%)$ sowie aus dem ehemaligen Jugoslawien (11,5\%) (Baier \& Pfeiffer 2007, 19).

Während die Jugenddelinquenz - auch bei Gewaltdelikten - nach den vorliegenden Dunkelfelduntersuchungen insgesamt tendenziell rückläufig ist, gibt es auch für Jugendliche mit Migrationshintergrund alles in allem keine 
Hinweise auf eine Zunahme delinquenten Verhaltens. Die Befunde deuten derzeit jedoch durchaus auf im Vergleich zu Einheimischen erhöhte Delinquenzrisiken in dieser Gruppe. Allerdings betrifft dies bei weitem nicht alle Deliktsbereiche, und stereotype Verallgemeinerungen verbieten sich schon deshalb, weil Jugendliche mit wiederholter Begehung zumal schwerer (Gewalt-)Delikte auch unter jungen männlichen Migranten eine absolute Minderheit darstellen. Die nicht immer konsistenten Ergebnisse in den verschiedenen Erhebungsregionen weisen zudem auf eine nicht unerhebliche Bedeutung des spezifischen städtischen Kontextes sowie der Größe und Zusammensetzung der jeweiligen Migrantenpopulation für die Delinquenzbelastung unter Jugendlichen mit Migrationshintergrund hin.

\section{Erklärungsansätze zur Delinquenz unter Jugendlichen ausländischer Herkunft}

Nach den empirischen Befunden kann es bei der Diskussion um Erklärungsansätze zu delinquentem Verhalten unter jungen Migranten nicht pauschal darum gehen, nach Jugenddelinquenz verstärkenden Auswirkungen von Migrationserfahrungen zu suchen. So ist es wenig sinnvoll, spezifische Ursachen für die allem Anschein nach „gewöhnliche“, jugendtypische Belastung junger Migranten bei leichterer Eigentums- und Sachbeschädigungsdelinquenz auszumachen. Umgekehrt erscheint es vielmehr erklärungsbedürftig, warum hier zum Teil, gerade bei weiblichen Jugendlichen aus bestimmten Migrantengruppen, eine unterdurchschnittliche Belastung beobachtet wird. Mögliche Delinquenz hemmende Faktoren in bestimmten Migrationskonstellationen werden indes bislang kaum diskutiert.

Gerade nach sozialstrukturellen Kriminalitätstheorien müssen Jugendliche aus bestimmten Migrantengruppen jedoch als „besonders gefährdet" gelten. Vor dem Hintergrund sozioökonomischer Benachteiligungen und schlechterer beruflicher Perspektiven - trotz im Vergleich mit ihrer Elterngeneration durchaus beobachtbaren Bildungsfortschritten erwerben Jugendliche mit Migrationshintergrund im Schnitt deutlich niedrigere Bildungsabschlüsse als ihre autochthonen Mitschüler/ innen - wird anomietheoretisch häufig auf die Spannungen zwischen den gesellschaftlich vermittelten Zielen und den zu deren Erreichung verfügbaren Mitteln hingewiesen, denen Jugendliche ausländischer Herkunft in besonderem Maße ausgesetzt seien (siehe etwa Geißler 2002, 38 sowie Tonry 1997, 21). Ein bei Angehörigen der zweiten Einwanderergeneration stärker ausgeprägtes Empfinden so- zialer Benachteiligungen soll danach auch erklären, warum die Nachkommen von Migranten eher wegen Kriminalität auffallen als die Einwanderer selbst. Einflüsse des sozialen Status auf Jugenddelinquenz haben sich in der Vergangenheit am ehesten indirekt und insbesondere dann gezeigt, wenn dabei schwerere Formen von Delinquenz sowie der soziale Status der Jugendlichen selbst (und nicht etwa die Schichtzugehörigkeit ihrer Eltern) berücksichtigt wurden. Im Hinblick auf die Gewaltbelastung von Migrantenjugendlichen relativieren sich die Unterschiede gegenüber jungen Einheimischen regelmäßig, wenn die Bildungsbeteiligung als sozialstruktureller Indikator kontrolliert wird - junge Migranten sind also nicht wesentlich auffälliger als einheimische Jugendliche mit gleicher Bildungsbeteiligung (vgl. etwa Naplava 2005, 61 ff.; Walburg 2007, 260 ff.). Allerdings verschwinden die Unterschiede dabei nicht immer vollständig (so etwa bei Wilmers et al. 2002, 183 ff. sowie bei Fuchs et al. 2005, 202).

Neben der Ungleichheit im Bildungsbereich macht auch ein vermehrtes Aufwachsen junger Migranten in unterprivilegierten Stadtvierteln als sozialräumlicher Kontexteffekt, der sich aus einer geringen kollektiven sozialen Kontrolle bzw. mangelndem sozialem Kapital sowie aus subkulturellen Lernprozessen ergeben könnte, erhöhte Delinquenzraten plausibel. In der sozialen Desorganisation in bestimmten Stadtvierteln (und nicht in der Einwanderung selbst oder der ethnischen Herkunft) sahen schon Shaw und McKay (1942) den wesentlichen Grund für Delinquenz unter jungen Migranten im Chicago des frühen 20. Jahrhunderts. Oberwittler (2004) konnte in einer Mehrebenenanalyse bei einheimischen Jugendlichen zusätzliche, das heißt von der individuellen sozialen Lage unabhängige Auswirkungen der räumlichen Konzentration sozialer Benachteiligung auf schwere Delinquenz bestätigen. Allerdings zeigten sich in seiner Untersuchung bei Jugendlichen mit Migrationshintergrund diesbezüglich etwas überraschend gerade keine oder sogar entgegengesetzte Zusammenhänge.

Ein besonderes Gewicht wird derzeit, häufig unter dem Oberbegriff der Kulturkonflikttheorie, solchen Erklärungsansätzen beigemessen, die die Bedeutung von kulturellen Unterschieden bzw. Modernitätsdifferenzen zwischen Herkunfts- und Aufnahmegesellschaft für die Lebenssituation von Migranten hervorheben und die daraus möglicherweise resultierenden Belastungen und Konflikte in einen Zusammenhang mit Delinquenz bringen. Im einfachsten Fall befolgen dabei Einwanderer Normen des Herkunftslandes und verstoßen da- durch unmittelbar gegen Normen der Aufnahmegesellschaft. Derartige Konstellationen - schon bei Sellin $(1938,68)$ findet sich dazu das Beispiel des sizilianischen Einwanderers in New Jersey, der zur Verteidigung der Familienehre den Verführer seiner Tochter tötet - dürften allerdings insgesamt und jenseits bestimmter Aufsehen erregender Ausnahmefälle von eher geringer Bedeutung für delinquentes Verhalten unter Jugendlichen sein (Walter 2005, 141 f.). Auch die Beobachtung, wonach die jeweils erste Migrantengeneration, die tendenziell stärker durch die Herkunftskultur geprägt sein müsste als ihre Nachkommen, eher unterdurchschnittliche Delinquenzraten aufweist, spricht gegen derlei Annahmen. Diese stoßen überdies auf grundsätzliche Kritik, insoweit sie von der Existenz homogener, statischer, klar abgrenzbarer und unvereinbarer Kulturen ausgehen, durch die die jeweiligen Angehörigen noch dazu weitgehend determiniert sind (siehe etwa Bukow \& Llaryora 1988). Dies erscheint in Bezug auf Migrantenjugendliche der zweiten bzw. dritten Generation umso mehr fragwürdig, als diese bereits in der Aufnahmegesellschaft sozialisiert wurden. Nichtsdestoweniger dominiert die Vorstellung, dass beispielsweise gewalttätiges Verhalten unter manchen türkischstämmigen Jugendlichen oder jungen Aussiedlern in erster Linie auf deren „Türkisch-“ bzw. „Russischsein“ zurückzuführen ist, das Alltagsverständnis und den öffentlichen Diskurs (Spindler 2006, 98 f.).

Anknüpfungspunkte für derartige Interpretationen liefern Befunde, wonach gewaltakzeptierende, an traditionellen Geschlechterrollen und Ehrbegriffen orientierte Normen gerade bei jungen Migranten türkischer Herkunft, aber auch bei Jugendlichen aus dem ehem. Jugoslawien sowie aus der ehemaligen Sowjetunion überdurchschnittlich weit verbreitet sind (siehe zuletzt beispielsweise Baier \& Pfeiffer 2007). Es ist jedoch fraglich, ob die Akzeptanz solcher Normen tatsächlich allein mit der familiären Herkunft aus ländlichen, „vormodernen“ Gesellschaften zu erklären ist. Bei der Analyse kultureller Prägungen sollten die strukturellen Bedingungen in der Aufnahmegesellschaft nicht aus dem Blick geraten: Danach könnten „traditionelle“ Vorstellungen von Männlichkeit und damit einhergehende Gewalteinstellungen zumindest auch als Folge von Deprivationserfahrungen und Marginalisierungsprozessen im Aufnahmeland zu deuten sein (Enzmann et al. 2004, 283 ff.; Halm 2000, 291). In diesem Zusammenhang kann gewaltaffine Maskulinität für männliche Jugendliche mit anderweitig schwach ausgeprägten Ressourcen, denen ein Rückgriff auf anerkannte Formen von Männlichkeit versagt bleibt, unabhängig von deren „kultureller“ 
oder „ethnischer“ Herkunft eine identitätsstiftende Funktion haben (Kersten 2003; Spindler 2006; Fuchs et al. 2005, 42 f.). Gestützt wird diese Überlegung durch die Beobachtung, dass eine Akzeptanz dieser Normen gerade nicht auf bestimmte ethnisch definierte Gruppen beschränkt ist (Enzmann et al. 2004, 283) und Jugendliche deutscher und sonstiger Herkunft unter vergleichbaren Lebensbedingungen ähnliche Männlichkeitsbilder und Gewaltpotenziale herausbilden.

Als eher indirekte Folge kultureller Dissonanzen sowie der von Fremdheits- und Ablehnungserfahrungen begleiteten gesellschaftlichen Positionierung werden Identitätsprobleme diskutiert, die bei jungen Migranten möglicherweise über die typischerweise im Jugendalter auftretenden Verunsicherungen und Orientierungsschwierigkeiten hinausgehen können. So überzeugend das Bild des „marginal man" vor allem aus Sicht der Mehrheitsgesellschaft erscheint, so sehr würde jedoch ein einseitiges Bild des zwischen den Kulturen gefangenen, entwurzelten und haltlosen Migrantenkindes den häufig flexiblen, als Normalität empfundenen transnationalen Biographie-Arrangements vieler Nachkommen von Einwanderern nicht gerecht (Beck-Gernsheim 2004, 74 ff.). Überdies können auch Identitätsprobleme mit strukturellen Bedingungen zusammenhängen, die mit kulturellen Differenzen an sich überhaupt nichts zu tun haben. $\mathrm{Zu}$ denken ist etwa an die speziellen Folgen von Fluchterfahrungen sowie an die (zum Teil massiven) ökonomischen, sozialen und rechtlichen Restriktionen, unter denen ein Teil der Einwanderer lebt (Spindler \& Tekin 2003, 216 ff.).

Im Zusammenhang mit kulturellen Divergenzen wird schließlich auf besondere Belastungen für das Eltern-Kind-Verhältnis im Migrationskontext verwiesen, die aus einer Art migrantenspezifischem Generationenkonflikt zwischen den Eltern und ihren stärker den kulturellen Einflüssen der Aufnahmegesellschaft ausgesetzten Kindern herrühren und eine Minderung der elterlichen Kontrolle sowie einen größeren Einfluss von (möglicherweise delinquenten) Gleichaltrigengruppen zur Folge haben können (vgl. etwa Schneider 1994, 40 f.). Auch diesbezüglich erscheint es jedoch wenig angemessen, jede Auseinandersetzung zwischen zugewanderten Eltern und ihren Kindern als migrationsbedingten Kulturkonflikt $\mathrm{zu}$ interpretieren und Migrantenfamilien allein als potentiellen Ort von Defiziten und Konflikten wahrzunehmen. So könnten die vielfach postulierten „traditionelleren“ und mit größeren (auch emotionalen) familiären Bindungen einhergehenden Orientierungen in bestimmten Migrantengruppen (zu Migranten türkischer Herkunft siehe etwa Heitmeyer et al. 1997, 70 ff.), die unter anderem eine Antwort auf Brüche und Unsicherheiten in der Migrationssituation sowie auf Ablehnungserfahrungen in der Aufnahmegesellschaft sein können, kriminologisch durchaus kompensierend wirken (Lösel \& Bliesener 2003, 85). Gerade die zum Teil zu beobachtende unterdurchschnittliche Delinquenzbelastung unter bestimmten weiblichen Migrantenjugendlichen gibt Anlass dazu, die familiären Sozialisationsbedingungen eingehender darauf $\mathrm{zu}$ untersuchen, inwiefern kriminologisch eher sogar eine geschlechtsspezifisch größere elterliche Kontrolle sowie entsprechende Konformitätsanforderungen zum Tragen kommen. So ergaben sich aus den Befragungen unter türkischstämmigen Jugendlichen in Duisburg Zusammenhänge zwischen einem weniger nach außen orientierten, mit seltenerem abendlichem Ausgehen etc. verbundenen Lebensstil und geringerer Delinquenz.

Als weiterer Delinquenz hemmender Umstand ließ sich dort, sowohl unter weiblichen als auch unter männlichen Jugendlichen türkischer Herkunft, ein im Vergleich zu einheimischen Mitschüler/innen deutlich geringerer Konsum von Alkohol beobachten. Darüber hinaus ging eine größere Zustimmung zu „traditionellen" Familienbildern und Geschlechterrollen, die hier als allgemeine Wertorientierungen und nicht als Einstellungen zu Gewalt erfragt wurden, für sich genommen ebenso wenig mit vermehrtem gewaltsamen und sonstigen delinquenten Verhalten einher wie eine deutlich größere Religiosität (vgl. hierzu, auf Grundlage der Befragungen des KFN, Brettfeld \& Wetzels 2004). Zugleich deuteten die Antworten der Duisburger Jugendlichen mit Migrationshintergrund nicht auf eine generell schlechtere Eltern-Kind-Beziehung. Allerdings ließ sich wie bereits in anderen Untersuchungen beobachten, dass gerade Jugendliche türkischer Herkunft zwar nur zu einem kleineren Teil, aber doch deutlich häufiger als einheimische Jugendliche einem gewaltsamen Erziehungsstil ausgesetzt sind, welcher seinerseits mit eigenem Gewalthandeln korreliert (vgl. auch Baier \& Pfeiffer 2007).

\section{Ausblick}

Delinquenz ist auch unter Migrantenjugendlichen in erster Linie jugendtypischer Bestandteil des Sozialisationsprozesses, und damit ein vielfach passageres Phänomen, das in den meisten Fällen ohne formelle Kontrollintervention abbricht. Bei darüber hinausgehenden Aussagen über besondere Delinquenz- risiken unter Jugendlichen mit Migrationshintergrund besteht schnell die Gefahr einer unzulässigen Pauschalisierung und entsprechender Fehlschlüsse. Die Migrationskonstellationen sind zunehmend vielgestaltig, und junge Einwanderer oder Kinder von Einwanderern wachsen unter den unterschiedlichsten Bedingungen auf. Die Ausblendung der Heterogenität, so typisch sie für die Wahrnehmung von „Fremden“ ist, und damit die Übertragung eines Merkmals auf eine gesamte (sozial konstruierte) Gruppe ist im Hinblick auf Kriminalität besonders folgenreich, ist doch kaum etwas so sehr geeignet, andere abzuwerten wie die Kategorie des Kriminellen.

Trotz der aufgezeigten Probleme einer genauen Quantifizierung lässt sich wohl sagen, dass ein überdurchschnittlich hoher Anteil der Jugendlichen, die wegen einer Straftat registriert werden, eigene oder familiäre Migrationserfahrungen hat. Es gibt Hinweise darauf, dass hierzu ein erhöhtes Kriminalisierungsrisiko von als fremd oder ausländisch wahrgenommenen Jugendlichen beiträgt. Befragungen unter Schüler/innen zeigen einerseits, dass auch unter Jugendlichen ausländischer Herkunft leichtere Formen von Delinquenz im Vordergrund stehen, welche ihrerseits nicht häufiger vorkommen als unter einheimischen Jugendlichen. Eine Reihe von Studien ergaben andererseits bei Gewaltdelikten sowie bei Berücksichtigung der Intensität eine erhöhte Delinquenzbelastung. Eine Suche nach Gründen hierfür wird die angesprochene Heterogenität der Lebensumstände zu berücksichtigen haben, es kommen darin jedoch problematische Sozialisationsbedingungen bei einem Teil der (vor allem männlichen) Migrantenjugendlichen zum Ausdruck. Klar belegt ist etwa die Auswirkung der Bildungsbenachteiligung, wobei die dabei zum Tragen kommenden Mechanismen offen sind. Auch soweit sich Hinweise auf problematische Gewalteinstellungen ergeben, bedarf es einer genaueren Analyse der Entstehungszusammenhänge; eine einseitige Interpretation als ethnisch-kulturell verwurzelte Wertorientierungen greift sicherlich $\mathrm{zu}$ kurz. Künftige Untersuchungen werden möglicherweise näher beleuchten können, ob und unter welchen Bedingungen die Migrationssituation problematische Lebenslagen darüber hinaus kriminologisch sogar abzufangen vermag und inwieweit sich im Generationenvergleich - insbesondere vormalige Gastarbeiterfamilien leben zum Teil in der vierten Generation in Deutschland - Veränderungen ergeben.

Christian Walburg, Doktorand am Institut für Kriminalwissenschaften, Abteilung Kriminologie der Universität Münster. 


\section{Literatur}

Baier, D. \& Pfeiffer, C. (2007): Gewalttätigkeit bei deutschen und nichtdeutschen Jugendlichen - Befunde der Schülerbefragung 2005 und Folgerungen für die Prävention. KFN-Forschungsbericht Nr. 100. Hannover: KFN.

Beck-Gernsheim, E. (2004). Wir und die Anderen. Vom Blick der Deutschen auf Migranten und Minderheiten. Frankfurt/Main: Suhrkamp.

Boers, K., Walburg, C. \& Reinecke, J. (2006). Jugendkriminalität - Keine Zunahme im Dunkelfeld, kaum Unterschiede zwischen Einheimischen und Migranten. Befunde aus Duisburger und Münsteraner Längsschnittstudien. Monatsschrift für Kriminologie und Strafrechtsreform, 89, 63-87.

Brettfeld, K. \& Wetzels, P. (2004). Junge Muslime in Deutschland: Eine kriminologische Analyse zur Alltagsrelevanz von Religion und Zusammenhängen von individueller Religiosität mit Gewalterfahrungen, -einstellungen und -handeln. In: Bundesministerium des Innern (Hg.), Texte zur Inneren Sicherheit. Islamismus, 221-316. 4. Aufl. Berlin.

Bukow, W.-D. \& Llaryora, R. (1988). Mitbürger aus der Fremde. Soziogenese ethnischer Minoritäten. Opladen: Westdeutscher Verlag.

Elias, N. \& Scotson, J. L. (1990): Etablierte und Außenseiter. Frankfurt/Main: Suhrkamp.

Enzmann, D., Brettfeld, K. \& Wetzels, P. (2004). Männlichkeitsnormen und die Kultur der Ehre. Empirische Prüfung eines theoretischen Modells zur Erklärung erhöhter Delinquenzraten jugendlicher Migranten. Sonderheft der Kölner Zeitschrift für Soziologie und Sozialpsychologie, 43/2003, 264-287.

Fuchs, M., Lamnek, S., Luedtke, J. \& Baur, N. (2005). Gewalt an Schulen. 1994 - 1999 - 2004. Wiesbaden: VS Verlag für Sozialwissenschaften.

Geißler, R. (2002). „Ausländerkriminalität“ - Vorurteile, Missverständnisse, Fakten. Anmerkungen zu einer vielschichtigen Problematik. In G. Kawamura-Reindl, R. Keicher \& W. Krell (Hg.), Migration, Kriminalität und Kriminalisierung. Herausforderung an Soziale Arbeit und Straffälligenhilfe, 2745. Freiburg i. Br: Lambertus.

Grundies, V. (2000). Kriminalitätsbelastung junger Aussiedler. Ein Längsschnittvergleich mit in Deutschland geborenen jungen Menschen anhand polizeilicher Registrierungen. Monatsschrift für Kriminologie und Strafrechtsreform, 83, 290305

Halm, D. (2000). Tradition, soziale Ungleichheit und Devianz. Thesen zu den Ursachen erhöhter Gewaltneigung junger Männer mit Migrationshintergrund. Kriminologisches Journal, 32, 286-292.

Heitmeyer, W. (1995). Deutsche und ausländische Jugendliche. Zur Brisanz ethnisch-kultureller Gewaltpotentiale. In W. Heitmeyer, B. Collmann, J. Conrads, I. Matuschek, D. Kraul, W. Kühnel, R. Möller \& M. Ulbrich-Hermann, Gewalt. Schattenseiten der Individualisierung bei Jugendlichen aus unterschiedlichen Milieus, 399-409. Weinheim und München: Juventa.

Heitmeyer, W., Müller, J. \& Schröder, H. (1997). Verlockender Fundamentalismus. Türkische Jugendliche in Deutschland. Frankfurt a.M.: Suhrkamp.
Kersten, J. (2003). „Gender and Crime“. Die Tragweite kulturübergreifender Ansätze. In S. Lamnek \& M. Boatcă (Hg.), Geschlecht - Gewalt - Gesellschaft, 71-84. Opladen: Leske und Budrich.

Kleimann, M. \& Pfeiffer, C. (2004). Zur Kriminalität junger Aussiedler - Aktuelle Befunde und Erklärungsansätze. Zeitschrift für Jugendkriminalrecht und Jugendhilfe, 15 , 378-384.

Konsortium Bildungsberichterstattung (Hg.). (2006). Bildung in Deutschland. Ein indikatorengestützter Bericht mit einer Analyse zu Bildung und Migration. Bielefeld: Bertelsmann.

Lösel, F. \& Bliesener, T. (2003). Aggression und Delinquenz unter Jugendlichen. Untersuchungen von kognitiven und sozialen Bedingungen. München, Neuwied: Luchterhand.

Luff, J. (2000). Kriminalität von Aussiedlern: Polizeiliche Registrierungen als Hinweis auf misslungene Integration? München: KFG, Bayerisches Landeskriminalamt.

Mansel, J. (2001). Angst vor Gewalt. Eine Untersuchung zu jugendlichen Opfern und Tätern. Weinheim und München: Juventa.

Mansel, J. \& Albrecht, G. (2003). Die Ethnie des Täters als ein Prädiktor für das Anzeigeverhalten von Opfern und Zeugen. Die private Strafanzeige als Form der Konfliktregulierung. Soziale Welt, 54, 339-372.

Mansel, J. \& Hurrelmann, K. (1998). Aggressives und delinquentes Verhalten Jugendlicher im Zeitvergleich. Befunde der "Dunkelfeldforschung" aus den Jahren 1988, 1990 und 1996. Kölner Zeitschrift für Soziologie und Sozialpsychlogie, 50, 78-109.

Naplava, T. (2003). Selbstberichtete Delinquenz einheimischer und immigrierter Jugendlicher im Vergleich. Eine Sekundäranalyse von Schulbefragungen der Jahre 1995-2000. Soziale Probleme, $14,67-96$.

Naplava, T. (2005): Jugenddelinquenz im interethnischen Vergleich. Erklärungsmöglichkeiten delinquenten Verhaltens einheimischer und immigrierter Jugendlicher. Dissertation. Bielefeld.

Oberwittler, D. (2004). Stadtstruktur, Freundeskreise und Delinquenz. Eine Mehrebenenanalyse zu sozialökologischen Kontexteffekten auf schwere Jugenddelinquenz. Sonderheft der Kölner Zeitschrift für Soziologie und Sozialpsychologie, 43/2003, 135-170.

Oberwittler, D., Blank, T., Köllisch, T. \& Naplava T. (2001). Soziale Lebenslagen und Delinquenz von Jugendlichen: Ergebnisse der MPI-Schulbefragung 1999 in Freiburg und Köln. Freiburg i. Br.: edition iuscrim.

Ohder, C. \& Huck, L. (2006): „Intensivtäter“ in Berlin - Hintergründe und Folgen vielfacher strafrechtlicher Auffälligkeit - Teil 1 Eine Auswertung von Akten der Abteilung 47 der Berliner Staatsanwaltschaft. In: Landeskommission Berlin gegen Gewalt (Hg.), Berliner Forum Gewaltprävention, Nr. 26, 6-57. Berlin.

Othold, F. \& Schumann, K. (2003). Delinquenzverläufe nach Alter, Geschlecht und Nationalitätenstatus. In K. Schumann (Hg.), Delinquenz im Lebensverlauf. Bremer Längsschnittstudie zum Übergang von der Schule in den Beruf bei ehema- ligen Hauptschülern. Band 2, 67-94. Weinheim und München: Juventa.

Pfeiffer, C., Kleimann, M., Petersen, S. \& Schott, T. (2005). Migration und Kriminalität. Ein Gutachten für den Zuwanderungsrat der Bundesregierung. Baden-Baden: Nomos.

Pfeiffer, C. \& Dworschak, B. (1999): Die ethnische Vielfalt in den Jugendvollzugsanstalten. Ergebnisse einer Umfrage aus dem Sommer 1998. DVJJJournal 10(2), 184-187.

Polizeipräsident in Berlin (Hg.). (2006). Polizeiliche Kriminalstatistik 2005. Berlin.

Schneider, H. J. (1994). Kriminologie der Gewalt. Suttgart, Leipzig: Hirzel.

Sellin, T. (1938). Culture Conflict and Crime. New York: Social Science Research Council.

Shaw, C. R. \& McKay, H. D. (1942). Juvenile Delinquency and Urban Areas: A Study of Rates of Delinquents in Relation to Differential Characteristics of local Communities in American Cities. Chicago: The University of Chicago Press.

Spindler, S. (2006). Corpus delicti. Männlichkeit, Rassismus und Kriminalisierung im Alltag jugendlicher Migranten. Münster: Unrast. (Zugl. Dissertation an der Universität zu Köln 2005)

Spindler, S. \& Tekin, U. (2003). Flüchten und sitzen bleiben: Jugendliche im Migrationskontext. In W.D. Bukow, K. Jünschke, S. Spindler \& U. Tekin (Hg.), Ausgegrenzt, eingesperrt und abgeschoben. Migration und Jugendkriminalität, 215-237. Opladen: Leske und Budrich.

Strobl, R. \& Kühnel, W. (2000). Dazugehörig und ausgegrenzt. Analysen zu Integrationschancen junger Aussiedler. Weinheim und München: Juventa.

Tonry, M. (1997). Ethnicity, Crime, and Immigration. In ders. (Hg.), Ethnicity, Crime, and Immigration. Comparative and Cross-National Perspectives, 1-29. Chicago, London: The University of Chicago Press.

Walburg, C. (2007). Migration und selbstberichtete Delinquenz. In K. Boers \& J. Reinecke (Hg.), Delinquenz im Jugendalter. Erkenntnisse einer Münsteraner Längsschnittstudie, 241-268. Münster: Waxmann.

Walter, M. (2005). Jugendkriminalität. Eine systematische Darstellung. 3. Aufl. Stuttgart: Boorberg.

Wilmers, N., Enzmann, D., Schaefer, D., Herbers, K., Greve, W. \& Wetzels, P. (2002). Jugendliche in Deutschland zur Jahrtausendwende: Gefährlich oder gefährdet? Baden-Baden: Nomos. 